TITLE:

\title{
Species Diversity of Marine Sponges along Chanthaburi and Trat Provinces, the Eastern Coast of the Gulf of Thailand
}

\author{
AUTHOR(S): \\ PUTCHAKARN, SUMAITT
}

CITATION:

PUTCHAKARN, SUMAITT. Species Diversity of Marine Sponges along Chanthaburi and Trat Provinces, the Eastern Coast of the Gulf of Thailand. Publications of the Seto Marine Biological Laboratory 2011, 41: 17-23

\section{ISSUE DATE:}

2011

URL:

http://hdl.handle.net/2433/159486

RIGHT: 


\title{
Species Diversity of Marine Sponges along Chanthaburi and Trat Provinces, the Eastern Coast of the Gulf of Thailand
}

\author{
SUMAITT PUTCHAKARN
}

\begin{abstract}
Marine Biodiversity Research Unit, Institute of Marine Science, Burapha University Bangsaen, Chon Buri 20131 THAILAND Tel +66-38-391671 3 Fax +66-38-391674

E-mail address: sumaitt@bims.buu.ac.th ; sumaitt@hotmail.com
\end{abstract}

\begin{abstract}
Species diversity of marine sponges was investigated in various habitats along the coast and islands of Chanthaburi and Trat provinces during February to May 2006. Sample collection was conducted from 18 sites, mostly carried out by wading and SCUBA diving during daytime and the observations were randomly conducted in all collection sites. The results showed that 72 species from 11 orders, 37 families and 52 genera were identified. Out of these, three species were the new records from Thai Waters, namely Placospongia melobesioides, Eurypon sp. "black" and Rhabderemia sp. "brown". Most species were previously found in the Gulf of Thailand and the South China Sea. Species occurrence in various habitats was noted for some common sponges.
\end{abstract}

Key words: Marine sponges, Porifera, the Gulf of Thailand

\section{Introduction}

Chanthaburi and Trat provinces are located on the easternmost area of the eastern coast of the Gulf of Thailand (Fig. 1). These provinces play important roles as providing a nursery for both economically and non-economically important fauna. The coral reef system in this area is distinct from those of the other areas in the Gulf of Thailand in having barrier reef (Chao Loa reef) and

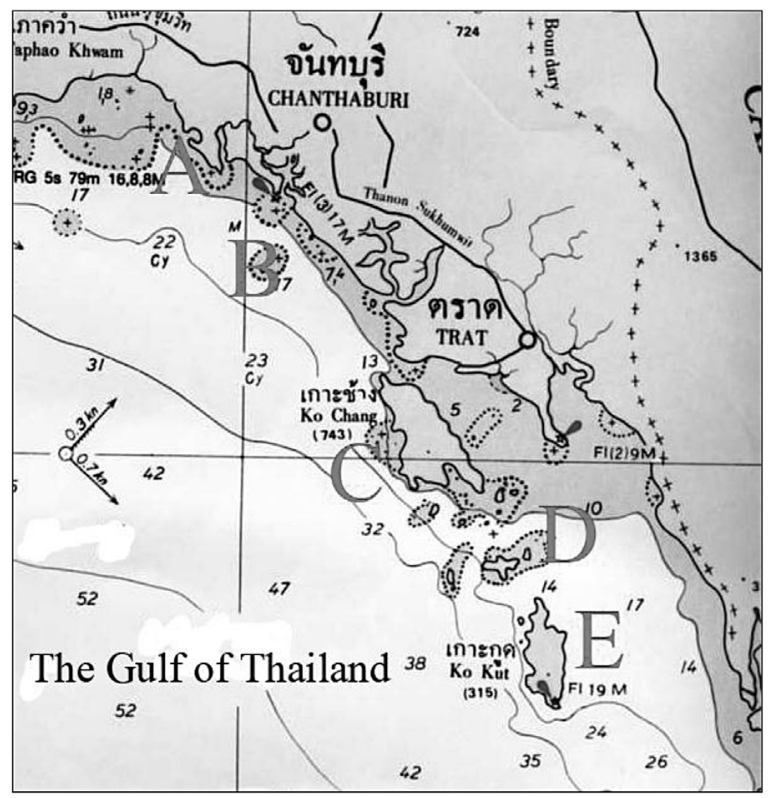

Fig. 1. Study area: Chanthaburi: A, Chao Loa reef; B, Ko Nom Soa, Trat: C, Ko Chang group, D, Ko Mak \& Ko Rung, E, Ko Kut group 
associated algae and seagrass beds (Ko Chang group). Putchakarn (2007) reviewed the literatures on sponges in the Gulf of Thailand and reported 45 demosponge species from the Had Khanom - Mo Ko Thale Tai National Park, in the southern part of the Gulf of Thailand. The purpose of the present study is to investigate the species diversity and distribution of marine sponges along the coast of Chanthaburi and Trat provinces to provide baseline study for subsequent investigations on the diversity and distribution of marine sponges in the Gulf of Thailand.

\section{Materials and Methods}

Collections. Sample collections were conducted in the coastal habitats along the Chanthaburi and Trat provinces, covering 18 sites of five locations (Fig. 1), during February to May 2006. Specimens were collected by wading and SCUBA diving during daytime, and observations were randomly made at all collection sites. The specimens were photographed in situ and notes were made based on morphological and ecological features such as colour, depth, and substrate. Specimens were preserved in 70\% alcohol and deposited at Institute of Marine Science, Burapha University, Thailand.

Laboratory work. Histological sections were made tangentially and perpendicularly to surface of the sponge with a scalpel. Sections were dried on a slide warmer and subsequently mounted in Canada balsam, and examined under a light microscope. Spicule preparations were made by putting fragment of sponge in boiling concentrated nitric acid, washed and centrifuged 3 times in distilled water and 3 times in $95 \%$ alcohol, and suspended in $95 \%$ alcohol. Spicule suspensions were pipetted onto microscopic glass slides, dried and mounted in Canada balsam for light microscopy. Spicules were measured based on 25 randomly chosen spicules for each category (Putchakarn et al., 2004; Putchakarn, 2006). Systematics follows Hooper and Van Soest (2002). Technical term follows Boury-Esnault and Rützler (1997).

\section{Results and Discussion}

During this study, 72 species (44 species to exact species level) of 52 genera, 37 families and 11 orders of class Demospongiae were found (Table 1 and Fig. 2). Of these, 29 species were reported from Chanthaburi province and 63 species from Trat province. Most species are common components in marine benthic habitats in the Gulf of Thailand and in the South China Sea (Chaitanawisuti et al., 2002; Hooper et al., 2000; Putchakarn, 2006, 2007). The most abundant and common sponges in this area were Xestospongia testudinaria (Lamarck) and Xestospongia sp. "purple" which were found in all locations, while Spheciospongia congenera (Ridley), Clathria (Thalysias) reinwardti Vosmaer, Gelliodes petrosioides Dendy, Neopetrosia sp. "blue", Dysidea arenaria Bergquist, Spongia sp., Hyrtios erectus (Keller) and Pseudoceratina sp. were found in four locations. Order Poecilosclerida (21 species) showed the highest species diversity, followed by Haplosclerida (18 species), while Xestospongia testudinaria and Xestospongia sp. "purple" (Haplosclerida) were the most common species. Three species from Chanthaburi and Trat provinces were the new records from Thai Waters, namely Placospongia melobesioides, Eurypon sp. "black" and Rhabderemia sp. "brown". Eight species were possibly new species: Plakina sp.1, Plakina sp.2, Raspailia (Raspaxilla) sp. "red", Eurypon sp. "black", Rhabderemia sp. "brown", Xestospongia sp. "purple", Dysidea sp. "blue" and Pseudoceratina sp. "yellow". Unfortunately these sponges were found to be rare species, mostly had small sample fragment and had not previously been studied in Thailand.

Several sponges from this study merit additional notes; Tetilla japonica Lampe appears to be especially found in sandy bottom offshore and somewhat turbid water areas of the eastern coast of Thailand. T. japonica is indicated as marine non-indigenous species in Thailand since type locality of this species was in Northern Japan (Chavanich et al., 2010; Lampe, 1886;). Plakina sp.1, Plakina sp.2, Ecionemia acervus Bowerbank, Rhabderemia sp. "brown", Placospongia melobesioides, Raspailia sp. "black" and Halichondria cartilaginea Esper were found only in Ko Chang group and needed further 


\section{SPECIES DIVERSITY OF MARINE SPONGES FROM THE EASTERN COAST OF THE GULF OF THAILAND}

Table 1. Species list and distribution of sponges from Chanthaburi and Trat provinces, the Gulf of Thailand

Study locations, Chanthaburi: 1, Chao Loa reef; 2, Ko Nom Soa, Trat: 3, Ko Chang group, 4, Ko Mak \& Ko Rung, 5, Ko Kut group

Distribution, X, present; -, absent

\begin{tabular}{|c|c|c|c|c|c|}
\hline \multirow{2}{*}{ Taxa } & \multicolumn{5}{|c|}{ Distribution } \\
\hline & 1 & 2 & 3 & 4 & 5 \\
\hline \multicolumn{6}{|l|}{ Class DEMOSPONGIAE Sollas, 1885} \\
\hline \multicolumn{6}{|l|}{ Order HOMOSCLEROPHORIDA Dendy, 1905} \\
\hline \multicolumn{6}{|l|}{ Family Plakinidae Schulze, 1880} \\
\hline 1. Plakina monolopha Schulze, 1880 & - & - & - & $\mathrm{X}$ & - \\
\hline 2. Plakina sp. 1 & - & - & - & $\mathrm{X}$ & - \\
\hline 3. Plakina sp. 2 & - & - & - & $\mathrm{X}$ & - \\
\hline \multicolumn{6}{|l|}{ Order SPIROPHORIDA Bergquist \& Hogg, 1969} \\
\hline \multicolumn{6}{|l|}{ Family Tetillidae Sollas, 1886} \\
\hline 4. Tetilla japonica Lampe, 1886 & - & - & - & $\mathrm{X}$ & - \\
\hline \multicolumn{6}{|l|}{ Order ASTROPHORIDA Sollas, 1888} \\
\hline \multicolumn{6}{|l|}{ Family Ancorinidae Schmidt, 1870} \\
\hline 5. Ecionemia acervus (Bowerbank, 1864 ) & - & - & $\mathrm{X}$ & - & - \\
\hline \multicolumn{6}{|l|}{ Family Calthropellidae Lendenfeld, 1907} \\
\hline 6. Pachastrissa nux (De Laubenfels, 1954) & - & - & $\mathrm{X}$ & - & - \\
\hline \multicolumn{6}{|l|}{ Family Geodiidae Gray, 1867} \\
\hline 7. Sidonops picteti Topsent, 1897 & - & - & $\mathrm{X}$ & - & - \\
\hline \multicolumn{6}{|c|}{ Order CHONDROSIDA Boury-Esnault \& Lopès, 1985} \\
\hline \multicolumn{6}{|c|}{ Family Chondrillidae Gray, 1872} \\
\hline 8. Chondrilla australiensis (Carter, 1883) & $\mathrm{X}$ & - & - & $\mathrm{X}$ & $\mathrm{X}$ \\
\hline 9. Chondrosia reticulata (Carter, 1886) & $\mathrm{X}$ & - & $\mathrm{X}$ & $\mathrm{X}$ & - \\
\hline \multicolumn{6}{|l|}{ Order HADROMERIDA Topsent, 1894} \\
\hline \multicolumn{6}{|l|}{ Family Clionaidae D’Orbigny, 1851} \\
\hline 10. Cliona aurivillii (Lindgren, 1897) & - & - & - & $\mathrm{X}$ & - \\
\hline 11. Spheciospongia congenera (Ridley, 1884) & $\mathrm{X}$ & - & $\mathrm{X}$ & $\mathrm{X}$ & $\mathrm{X}$ \\
\hline \multicolumn{6}{|l|}{ Family Placospongiidae Gray, 1867} \\
\hline 12. Placospongia melobesioides Gray, 1867 & - & - & - & $\mathrm{X}$ & - \\
\hline \multicolumn{6}{|l|}{ Family Spirastrellidae Ridley \& Dendy, 1886} \\
\hline 13. Spirastrellla solida (Ridley \& Dendy, 1886) & - & - & $\mathrm{X}$ & - & $\mathrm{X}$ \\
\hline \multicolumn{6}{|l|}{ Family Suberitidae Schmidt, 1870} \\
\hline 14. Terpios granulosa Bergquist, 1967 & - & - & $\mathrm{X}$ & $\mathrm{X}$ & - \\
\hline 15. Terpios sp. "yellow" & - & - & - & $\mathrm{X}$ & $\mathrm{X}$ \\
\hline 16. Terpios sp. "dark green" & - & - & - & $\mathrm{X}$ & - \\
\hline \multicolumn{6}{|l|}{ Family Tethyidae Gray 1848} \\
\hline 17. Tethya seychellensis (Wright, 1881) & - & - & - & - & $\mathrm{X}$ \\
\hline \multicolumn{6}{|l|}{ Family Timeidae Topsent, 1928} \\
\hline 18. Timea sp. "yellow" & - & - & $\mathrm{X}$ & $\mathrm{X}$ & - \\
\hline
\end{tabular}

Order POECILOSCLERIDA Topsent, 1928

Suborder MICROCIONINA Hajdu, Van Soest \& Hooper, 1994

Family Microcionidae Carter, 1875

19. Clathria (Microciona) aceratoobtusa (Carter, 1887)

20. Clathria (Microciona) sp. "orange"

21. Clathria (Thalysias) reinwardti Vosmaer, 1880

22. Clathria (Thalysias) toxifera (Hentschel, 1912)

23. Clathria (Thalysias) sp.

Family Raspailiidae Hentschel, 1923

24. Thrinacophora incrustans (Kieschnick, 1896)

25. Echinodictyum asperum Ridley \& Dendy, 1886

26. Eurypon sp. "black"

27. Hymeraphia sp. "red"

\begin{tabular}{ccccc}
\hline- & - & - & $\mathrm{X}$ & - \\
\hline $\mathrm{X}$ & - & $\mathrm{X}$ & $\mathrm{X}$ & - \\
\hline $\mathrm{X}$ & $\mathrm{X}$ & - & $\mathrm{X}$ & $\mathrm{X}$ \\
\hline- & $\mathrm{X}$ & - & - & - \\
\hline- & - & - & - & $\mathrm{X}$ \\
\hline & & & & \\
\hline- & - & $\mathrm{X}$ & $\mathrm{X}$ & - \\
\hline $\mathrm{X}$ & $\mathrm{X}$ & $\mathrm{X}$ & - & - \\
\hline- & - & $\mathrm{X}$ & - & - \\
\hline- & - & - & - & $\mathrm{X}$ \\
\hline
\end{tabular}


Table 1. Continued.

Study locations, Chanthaburi: 1, Chao Loa reef; 2, Ko Nom Soa, Trat: 3, Ko Chang group, 4, Ko Mak \& Ko Rung, 5, Ko Kut group

Distribution, X, present; -, absent

\begin{tabular}{|c|c|c|c|c|c|}
\hline \multirow{2}{*}{ Taxa } & \multicolumn{5}{|c|}{ Distribution } \\
\hline & 1 & 2 & 3 & 4 & 5 \\
\hline 28. Raspailia (Raspaxilla) sp. "red" & - & - & $\mathrm{X}$ & $\mathrm{X}$ & - \\
\hline 29. Raspailia sp. "black" & - & - & - & $\mathrm{X}$ & - \\
\hline Family Rhabderemiidae Topsent, 1928 & & & & & \\
\hline 30. Rhabderemia sp. "brown" & - & - & - & $\mathrm{X}$ & - \\
\hline $\begin{array}{l}\text { Suborder MYCALINA Hajdu, Van Soest \& Hooper, } 1994 \\
\text { Family Desmacellidae Ridley \& Dendy, } 1886\end{array}$ & & & & & \\
\hline 31. Biemna fortis (Topsent, 1897) & - & - & $\mathrm{X}$ & $\mathrm{X}$ & - \\
\hline Family Isodictyidae Dendy, 1924 & & & & & \\
\hline 32. Coelocarteria singaporensis (Carter, 1883) & - & - & - & - & $\mathrm{X}$ \\
\hline Family Mycalidae Lundbeck, 1905 & & & & & \\
\hline 33. Mycale (Mycale) grandis Gray, 1867 & - & - & $\mathrm{X}$ & - & $\mathrm{X}$ \\
\hline 34. Mycale (Zygomycale) parishii (Bowerbank, 1875) & $\mathrm{X}$ & - & $\mathrm{X}$ & $\mathrm{X}$ & - \\
\hline $\begin{array}{l}\text { Suborder MYXILLINA Hajdu, Van Soest \& Hooper, } 1994 \\
\text { Family Coelosphaeridae Dendy, } 1922\end{array}$ & & & & & \\
\hline 35. Lissodendoryx (Waldoschmittia) schmidti (Ridley, 1884) & - & - & - & - & $\mathrm{X}$ \\
\hline Family Hymedesmiidae Topsent, 1928 & & & & & \\
\hline 36. Phobas sp. "orange" & $\mathrm{X}$ & - & - & - & - \\
\hline Family Iotrochotidae Dendy, 1922 & & & & & \\
\hline 37. Iotrochota baculifera Ridley, 1884 & $\mathrm{X}$ & - & $\mathrm{X}$ & $\mathrm{X}$ & - \\
\hline Family Crambeidae Lévi, 1963 & & & & & \\
\hline 38. Monanchora unguiculata (Dendy, 1922) & - & - & - & $\mathrm{X}$ & $\mathrm{X}$ \\
\hline 39. Monanchora sp. "orange" & - & - & $\mathrm{X}$ & $\mathrm{X}$ & - \\
\hline $\begin{array}{l}\text { Order HALICHONDRIDA Gray, } 1867 \\
\text { Family Halichondriidae Gray, } 1867\end{array}$ & & & & & \\
\hline 40. Halichondria cartilaginea Esper, 1794 & - & - & $\mathrm{X}$ & - & - \\
\hline 41. Amorphinopsis siamensis (Topsent, 1925) & - & - & - & - & $\mathrm{X}$ \\
\hline Family Dictyonellidae Van Soest, Diaz \& Pomponi, 1990 & & & & & \\
\hline 42. Scopalina australiensis (Pulizer-Finali, 1982) & - & - & - & - & - \\
\hline $\begin{array}{l}\text { Order HAPLOSCLERIDA Topsent, } 1928 \\
\text { Suborder HAPLOSCLERINA Topsent, } 1928 \\
\text { Family Chalinidae Gray, } 1867\end{array}$ & & & & & \\
\hline 43. Haliclona (Halichoclona) sp. "white" & - & - & - & $\mathrm{X}$ & - \\
\hline 44. Haliclona (Halichoclona) sp. "yellow" & - & - & $\mathrm{X}$ & $\mathrm{X}$ & - \\
\hline 45. Haliclona (Halichoclona) sp. "purple" & - & - & $\mathrm{X}$ & - & - \\
\hline 46. Haliclona (Haliclona) sp. "brown" & - & - & - & - & $\mathrm{X}$ \\
\hline 47. Haliclona (Reniera) sp. "yellow" & $\mathrm{X}$ & $\mathrm{X}$ & - & - & - \\
\hline 48. Haliclona (Rhizoniera) sp. "blue" & - & - & - & $\mathrm{X}$ & $\mathrm{X}$ \\
\hline 49. Haliclona (Soestella) sp. "black" & $\mathrm{X}$ & $\mathrm{X}$ & - & - & - \\
\hline Family Callyspongiidae de Laubenfels, 1936 & & & & & \\
\hline 50. Callyspongia (Cladochalina) subarmigera Ridley, 1884 & - & - & - & - & $\mathrm{X}$ \\
\hline Family Niphatidae Van Soest, 1980 & & & & & \\
\hline 51. Amphimedon sp. & $\mathrm{X}$ & - & - & - & - \\
\hline 52. Dasychalina fragilis (Ridley \& Dendy, 1886) & - & - & $\mathrm{X}$ & $\mathrm{X}$ & $\mathrm{X}$ \\
\hline 53. Gelliodes petrosioides Dendy, 1905 & $\mathrm{X}$ & $\mathrm{X}$ & $\mathrm{X}$ & $\mathrm{X}$ & - \\
\hline $\begin{array}{l}\text { Suborder PETROSINA Boury-Esnault \& Van Beveren, } 1982 \\
\text { Family Petrosiidae Van Soest, } 1980\end{array}$ & & & & & \\
\hline 54. Neopetrosia sp. "blue" & $\mathrm{X}$ & $\mathrm{X}$ & $\mathrm{X}$ & $\mathrm{X}$ & - \\
\hline 55. Petrosia (Petrosia) hoeksemai De Voogd \& Van Soest, 2002 & $\mathrm{X}$ & $\mathrm{X}$ & - & - & - \\
\hline 56. Petrosia (Petrosia) sp. & - & - & - & - & $\mathrm{X}$ \\
\hline
\end{tabular}




\section{SPECIES DIVERSITY OF MARINE SPONGES FROM THE EASTERN COAST \\ OF THE GULF OF THAILAND}

Table 1. Continued.

Study locations, Chanthaburi: 1, Chao Loa reef; 2, Ko Nom Soa, Trat: 3, Ko Chang group, 4, Ko Mak \& Ko Rung, 5, Ko Kut group

Distribution, X, present; -, absent

\begin{tabular}{|c|c|c|c|c|c|}
\hline \multirow{2}{*}{ Taxa } & \multicolumn{5}{|c|}{ Distribution } \\
\hline & 1 & 2 & 3 & 4 & 5 \\
\hline 57. Xestospongia mamillata (Pultizer-Finali, 1981) & $\mathrm{X}$ & - & - & - & - \\
\hline 58. Xestospongia testudinaria (Lamarck, 1815) & $\mathrm{X}$ & $\mathrm{X}$ & $\mathrm{X}$ & $\mathrm{X}$ & $\mathrm{X}$ \\
\hline 59. Xestospongia sp. "purple" & $\mathrm{X}$ & $\mathrm{X}$ & $\mathrm{X}$ & $\mathrm{X}$ & $\mathrm{X}$ \\
\hline \multicolumn{6}{|l|}{ Family Phloeodictyidae } \\
\hline 60. Oceanapia sagittaria (Sollas, 1902) & $\mathrm{X}$ & - & $\mathrm{X}$ & $\mathrm{X}$ & - \\
\hline \multicolumn{6}{|l|}{$\begin{array}{l}\text { Order DICTYOCERATIDA Minchin, } 1900 \\
\text { Family Dysideidae Gray, } 1867\end{array}$} \\
\hline 61. Dysidea arenaria Bergquist, 1965 & $\mathrm{X}$ & - & $\mathrm{X}$ & $\mathrm{X}$ & $\mathrm{X}$ \\
\hline 62. Dysidea sp. "blue" & - & $\mathrm{X}$ & $\mathrm{X}$ & - & $\mathrm{X}$ \\
\hline 63. Lamellodysidea herbacea (Keller, 1889) & $\mathrm{X}$ & - & $\mathrm{X}$ & - & - \\
\hline \multicolumn{6}{|l|}{ Family Irciniidae Gray, 1867} \\
\hline 64. Ircinia mutans (Wilson, 1925) & $\mathrm{X}$ & - & - & $\mathrm{X}$ & - \\
\hline \multicolumn{6}{|l|}{ Family Spongiidae Gray, 1867} \\
\hline 65. Hyattella intestinalis (Lamarck, 1814) & $\mathrm{X}$ & - & - & - & - \\
\hline 66. Spongia sp. & $\mathrm{X}$ & $\mathrm{X}$ & $\mathrm{X}$ & $\mathrm{X}$ & - \\
\hline \multicolumn{6}{|l|}{ Family Thorectidae Bergquist, 1978} \\
\hline 67. Hyrtios erectus (Keller, 1889) & $\mathrm{X}$ & - & $\mathrm{X}$ & $\mathrm{X}$ & $\mathrm{X}$ \\
\hline \multicolumn{6}{|l|}{$\begin{array}{l}\text { Order DENDROCERATIDA Minchin, } 1900 \\
\text { Family Darwinellidae Mereikowsky, } 1879\end{array}$} \\
\hline 68. Aplysilla aff. rosea (Barrios, 1876) & - & - & - & - & $\mathrm{X}$ \\
\hline 69. Chelonaplysilla erecta (Row, 1911) & - & - & $\mathrm{X}$ & $\mathrm{X}$ & $\mathrm{X}$ \\
\hline \multicolumn{6}{|l|}{ Order VERONGIDA Bergquist, 1978} \\
\hline 70. Hexadella racovitzai Topsent, 1886 & $\mathrm{X}$ & - & $\mathrm{X}$ & - & $\mathrm{X}$ \\
\hline \multicolumn{6}{|l|}{ Family Pseudoceratinidae Carter, 1885} \\
\hline 71. Pseudoceratina purpurea (Carter, 1880$)$ & - & - & $\mathrm{X}$ & - & - \\
\hline 72. Pseudoceratina $\mathrm{sp}$. & $\mathrm{X}$ & - & $\mathrm{X}$ & $\mathrm{X}$ & $\mathrm{X}$ \\
\hline
\end{tabular}

studies. Mycale (Mycale) grandis Gray and M. (Zygomycale) parishii (Bowerbank) are common and widely distributed in the Gulf of Thailand. M. (Mycale) grandis is a burrowing sponge and inhabits crevices between the rocks or dead corals but $M$. (Zygomycale) parishii is an encrusting sponge and is usually associated with live bivalve shells or barnacles and occurs on dead gorgonians. Petrosia (Petrosia) hoeksemai De Voogd \& Van Soest has differential morphologies and is common in high sedimentation areas such as Chao Lao reef and Ko Nom Soa. Ircinia mutans (Wilson) also live in high sedimentation habitats such as on reef slope and on the outer reef in soft-bottom areas. Halichondria cartilaginea and Lamellodysidea herbacea (Keller) are common in the upper subtidal zone of coral reefs exposed to sunlight. Xestospongia sp. "purple" is thick encrusting sponge, usually found in the shaded area or in the cave of huge coral head.

\section{Acknowledgements}

This work was supported by the National Research Council of Thailand in the fiscal year 2006. Many thanks are due to Dr. Kitithorn Sanpanich and Mr. Sucha Munkongsomboon for help with sampling collection and to the director of research program, Dr. Chutiwan Decsakulwattana. I am indebted to Dr. Rob van Soest, Zoologisch Museum, University of Amsterdam, the Netherlands and Dr. Jane Fromont, Curator of Marine Invertebrates, Department of Aquatic Zoology, Western Australian Museum for consulting and helping on sponge taxonomic 


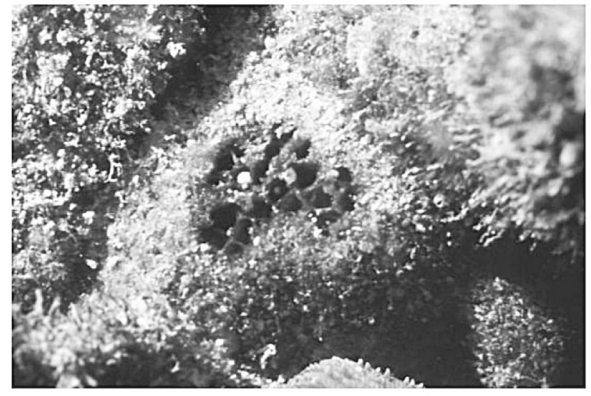

Ecionemia acervus (Bowerbank)

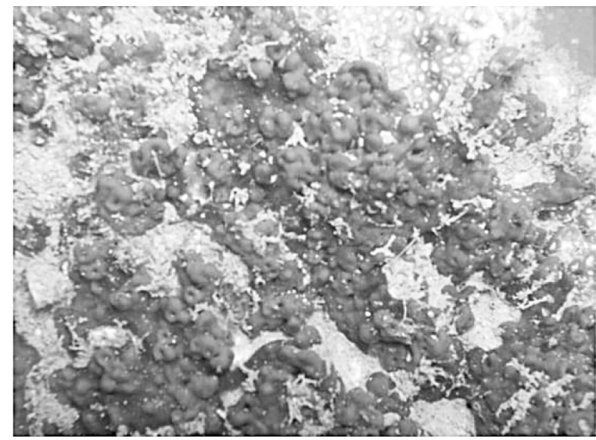

Rhabderemia sp. "brown"

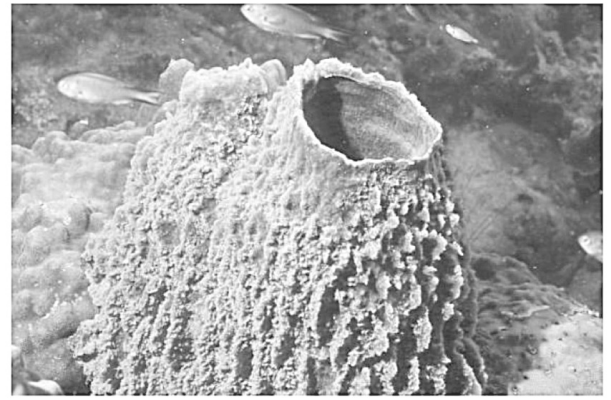

Xestospongia testudinaria (Lamarck)

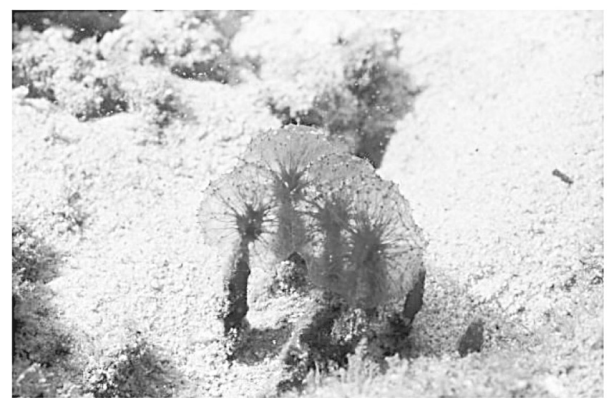

Oceanapia sagittaria (Sollas)

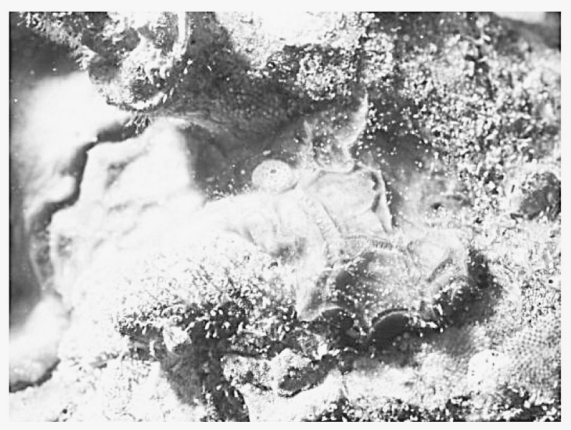

Placospongia melobesioides Gray

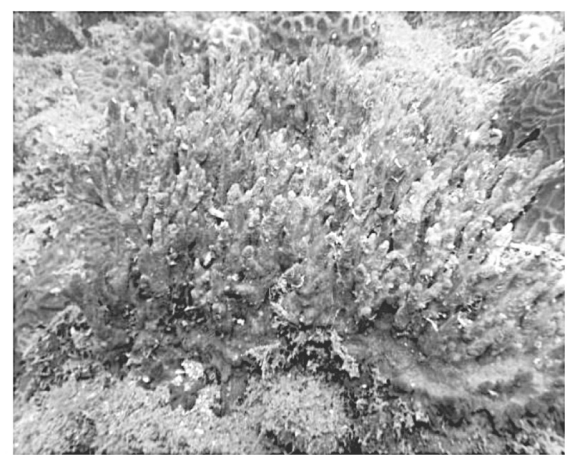

Halichondria cartilaginea Esper

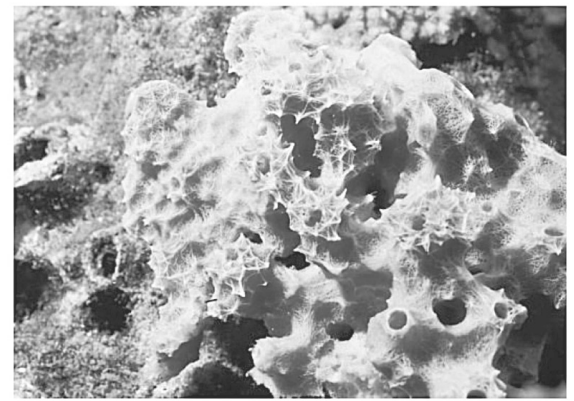

Dasychalina fragilis Ridley \& Dendy

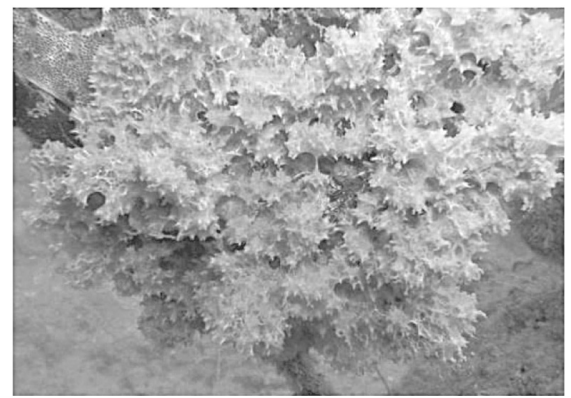

Dysidea arenaria Bergquist

Fig. 2. Underwater photos of some marine sponges from the Chantha-Buri and Trad provinces 


\section{SPECIES DIVERSITY OF MARINE SPONGES FROM THE EASTERN COAST \\ OF THE GULF OF THAILAND}

works. Finally, my close friend, Prof. Dr. Susumu Ohtsuka, Hiroshima University, who kindly supported the references for my request. Publication of this paper is financially supported in part by Natural Geography In Shore Areas (NaGISA) and Ministry of the Environment Japan (The Environment Research and Technology Development Fund S-9).

\section{References}

Boury-Esnault, N. and Rützler, K. 1997. Thesaurus of sponge morphology. Smithsonian Contributions to Zoology, $596,1-55$

Chaitanawisuti, N., Kritsanapuntu, S., Yeemin, T., Putchakarn, S., and Fromont J. 2002. Biodiversity of marine sponges associated with coral reef habitats in the eastern Gulf of Thailand (Chonburi to Trad Province). In Baimai V. and Tantalakha, R. (eds.) BRT Research Reports 2002, BRT Program: Chuan Printing, Bangkok, pp. 148-155.

Chavanich, S., Tan, L.T., Vallejo, B. and Viyakarn, V. 2010. Report on the current status of marine nonindigenous species in the Western Pacific region. Intergovernmental Oceanographic Commission SubCommission for the Western Pacific (IOC/WESTPAC), Bangkok, Thailand. $61 \mathrm{pp}$.

Hooper, J. N. A., Kennedy J. A. and Van Soest, R. W. M. 2000. Annotated Checklist of Sponges (Porifera) of the South China Sea Region. The Raffles Bulletin of Zoology, Supplement, no.8, 125-207.

Hooper, J. N. A. and Van Soest, R. W. M. 2002. Systema Porifera. vol. I., Kluwier Publisher Company, UK, 1101 pp.

Lampe, W. 1886. Eine neue Tetractinellidenform mit radiären Bau. Archiv für Naturgeschichte, 53, 1-18.

Putchakarn, S., De Weerdt, W., Sonchaeng, P. \& Van Soest, R.W.M. 2004. A new species of Cladocroce Topsent, 1892 (Porifera, Haplosclerida) from the Gulf of Thailand. Beaufortia, 54: 113-117.

Putchakarn, S. 2006. Biodiversity of sponges (Demonspongiae, Porifera) in the Gulf of Thailand. Ph.D. Thesis, Burapha University, Thailand.

Putchakarn, S. 2007. Species diversity of marine sponges dwelling in coral reefs in Had Khanom-Mo Ko Thale Tai National Park, Nakhon Si Thammarat Province, Thailand. Journal of the Marine Biological Association of the United Kingdom, 87, 1635-1642. 